\title{
Seed and biological productivity of Prunella vulgaris L. in situ and ex situ
}

\author{
Irina Barsukova ${ }^{1, *}$, and Alexei Astashenkov ${ }^{2}$ \\ ${ }^{1}$ N.F. Katanov, Khakasiya State University, 655017, Abakan, Russia \\ ${ }^{2}$ Central Siberian Botanical Garden SB RAS, 630090, Novosibirsk, Zolotodolinskaya str., 101, Russia
}

\begin{abstract}
Reproductive biology and biological productivity of Prunella vulgaris $\mathrm{L}$. were studied in natural growth conditions in Khakasiya and under introduction. It was established that in the wild a potential seed productivity of the species varied from 85,4 to 160,3 it./ a shoot, a real one - from 73,6 to 149,2 it.; erem germination under optimal conditions reached $90,5 \%$. Under introduction the indicators of seed productivity increase by $2-5$ times, of erem germination - by 1,5 times. Biological productivity of individuals in cultivation is by 5-10 times higher compared to that in natural habitats.
\end{abstract}

\section{Introduction}

Common selfheal (Prunella vulgaris L.) is a perennial herbaceous polycarpic plant with sympodially accreting rhizome. The species under study has a circumboreal range [1]. It is a valuable medicinal plant which posesses antimicrobic and antiviral activities [2]. In nature it is low-yielding. In this regard study of reproductive characteristics and biological productivity of $P$. vulgaris in the wild and under introduction will allow not only to judge adaptation of plants to the environment, but to obtain high-yielding crops. There is a considerable amount of data on reproductive biology of the species, but they encompass Europe and America [3-6]. In Siberian natural conditions the indicators of seed productivity of this species were studied only in Novosibirsk [7] and Tomsk [8] oblasts information on biological productivity is absent. The aim of the work was study and comparison of the pattern of reproductive biology and biological productivity of $P$. vulgaris in natural conditions of Khakasiya and under introduction.

\section{Material and methods}

Study of the indicators of reproductive biology of P. vulgaris was conducted in 2012-2014 in four coenopopulations located in different phytocoenoses of Khakasiya. Coenopopulation (CP) 1 is in bluegrass-orchard grass (Dactylis glomerata, Poa sibirica) forest meadow ; total projective cover degree (TPCD) $98 \%$, projective cover degree of the species (PCD) 5\%. CP 2 is in common lady's mantle (Alchemilla vulgaris) true meadow;

\footnotetext{
* Corresponding author: saphronovairina@mail.ru
} 
TPCD 95\%, PCD 10\%. CP 3 is in grasses-horsetail (Equisetum sylvaticum, Poa pratensis) forest meadow, TPCD $-65 \%$, PCD $-2 \%$. CP 4 is in orchard grass-aizoon stonecrop (Sedum aizoon, Dactylis glomerata) true meadow, TPCD - 45\%, PCD - 2\%. The introduction experiment was performed in Krasnoyrsk Krai. Under cultivation the pattern of reproductive biology was studied on individuals grown from erems collected in the same coenopopulations. Study of seed productivity was conducted by I. V. Vainagiy method [9]. Calculations were carried out on randomly selected ripe generative individuals. Germination pattern was also studied on the erems collected in the wild and under introduction. The erems of typical of $P$. vulgaris natural conditions of habitation (CP 2) were sown under winter to study biological productivity.

\section{Results and discussion}

The fruit of $P$. vulgaris is a coenobium, it consists of 4 fruitlets - erems of the combined type of dormancy [10]. P. vulgaris erems are fine, the mass of 1000 items in coenopopulations does not exceed $0,67 \pm 0,02 \mathrm{~g}$. Study of seed productivity indices in coenopopulations allowed to establish their similarity and dissimilarity depending on the year of study and phytocoenosis type (Table 1).

Table 1. Seed productivity of Prunella vulgaris individuals in natural habitats (per 1 generative shoot)

\begin{tabular}{|c|c|c|c|c|c|c|}
\hline № CP & Year & PSP, it. & $V^{*}, \%$ & RSP, it. & $\mathrm{V}, \%$ & Cp, $* * \%$ \\
\hline \multirow[t]{3}{*}{1} & 2012 & $\frac{160,32 \pm 9,59 * * *}{84-240}$ & 29,91 & $\frac{149,24 \pm 8,77}{76-229}$ & 29,38 & 93,1 \\
\hline & 2013 & $\frac{135,68 \pm 8,04}{72-216}$ & 29,65 & $\frac{122,40 \pm 8,12}{48-207}$ & 33,18 & 90,2 \\
\hline & 2014 & $\frac{125,76 \pm 6,54}{72-192}$ & 26,00 & $\frac{111,20 \pm 5,97}{62-186}$ & 26,86 & 88,4 \\
\hline \multirow[t]{3}{*}{2} & 2012 & $\frac{121,28 \pm 7,96}{72-192}$ & 32,82 & $\frac{110,76 \pm 7,22}{61-181}$ & 32,57 & 91,5 \\
\hline & 2013 & $\frac{100,80 \pm 6,04}{48-168}$ & 29,96 & $\frac{85,84 \pm 6,46}{29-142}$ & 37,62 & 85,2 \\
\hline & 2014 & $\frac{111,36 \pm 4,97}{48-168}$ & 22,33 & $\frac{101,08 \pm 4,65}{40-152}$ & 22,98 & 90,7 \\
\hline \multirow[t]{3}{*}{3} & 2012 & $\frac{134,88 \pm 8,59}{36-216}$ & 31,87 & $\frac{124,72 \pm 8,17}{28-204}$ & 32,77 & 92,4 \\
\hline & 2013 & $\frac{120,96 \pm 7,65}{48-192}$ & 31,63 & $\frac{109,44 \pm 6,82}{36-174}$ & 31,19 & 90,4 \\
\hline & 2014 & $\frac{111,52 \pm 11,95}{24-168}$ & 53,58 & $\frac{96,84 \pm 10,76}{16-156}$ & 55,58 & 86,8 \\
\hline \multirow[t]{3}{*}{4} & 2012 & $\frac{112,64 \pm 8,03}{36-168}$ & 35,67 & $\frac{103,76 \pm 7,61}{24-156}$ & 36,69 & 92,1 \\
\hline & 2013 & $\frac{85,44 \pm 6,17}{36-144}$ & 36,11 & $\frac{73,64 \pm 4,98}{27-111}$ & 33,83 & 86,2 \\
\hline & 2014 & $\frac{98,80 \pm 6,82}{24-144}$ & 34,47 & $\frac{87,24 \pm 5,50}{24-124}$ & 31,54 & 88,2 \\
\hline
\end{tabular}

Note: *Coefficient of variation; $* *$ coefficient of productivity $(\mathrm{Cp}) ; * * *$ above the line - average value \pm mistake of average value; below the line - minimal value - maximal value.

The highest values of potential seed productivity (PSP) were in all coenopopulations in 2012, low ones - in 2013 and 2014. The greatest values therewith were noted in 2012 in forest meadows, the lowest ones - in 2013 in true meadows. The distinctions established in PSP indices in individuals were linked with meteorological conditions of the vegetative 
season. Thus, prolonged moderate humidity and favourable warm weather from May to September in 2012 reflected positively on formation of the reproductive sphere compared to other years. Values of the real seed productivity (RSP) of P. vulgaris individuals regardless of habitat conditions were somewhat lower than PSP ones (Table 1), which was linked with not only the ecotope, but with high effectiveness of self-pollination directly affecting erem setting.

When introducing, increase in productivity by 2-5 times takes place independent of ecological-phytocoenotic species growth (Table 2). This is due to essential increase of the number of flowers in the main inflorescence (up to 78) on the shoots and in paracladia (up to 48). In addition, distinctions between reproductive indicators are determined by ontogenetic plant stage. Thus, the greatest values of PSP (up to 688 it.) and RSP (up to 76 it.) are observed in individuals at the ripe generative stage.

Of the relation between seed productivity of P. vulgaris and the number of generative shoots and inflorescences on them, as well as the number of flowers in the latter wrote A. A. Winn and P. A. Werner [5]. According to recent research conducted in Finland, the cutting regime may also positively affect reproductive characteristics of the species [11].

Table 2. Seed productivity of Prunella vulgaris individuals under introduction (per 1 generative shoot)

\begin{tabular}{|c|c|c|c|c|c|c|}
\hline \multirow{2}{*}{ № CP } & Year & PSP, it. & V,\% & RSP, it. & V,\% & Cp, $\%$ \\
\hline \multirow{2}{*}{$\mathbf{1}$} & 2012 & $\frac{366,72 \pm 11,29}{264-480}$ & 15,39 & $\frac{352,92 \pm 10,90}{240-457}$ & 15,45 & 96,2 \\
\cline { 2 - 7 } & 2013 & $\frac{499,64 \pm 18,23}{336-648}$ & 18,24 & $\frac{493,52 \pm 17,91}{331-631}$ & 18,15 & 98,7 \\
\hline \multirow{2}{*}{$\mathbf{2}$} & 2012 & $\frac{308,48 \pm 9,93}{216-384}$ & 16,11 & $\frac{297,88 \pm 9,47}{198-359}$ & 15,90 & 96,6 \\
\cline { 2 - 7 } & 2013 & $\frac{547,84 \pm 13,01}{444-688}$ & 11,87 & $\frac{537,24 \pm 13,05}{436-676}$ & 12,14 & 98,0 \\
\cline { 2 - 7 } & 2014 & $\frac{318,68 \pm 12,61}{144-408}$ & 19,78 & $\frac{299,56 \pm 12,56}{136-388}$ & 20,96 & 94,0 \\
\hline \multirow{3}{*}{$\mathbf{3}$} & 2012 & $\frac{306,40 \pm 9,88}{192-384}$ & 16,13 & $\frac{295,44 \pm 10,02}{187-374}$ & 16,97 & 96,4 \\
\cline { 2 - 7 } & 2013 & $\frac{463,84 \pm 16,78}{336-624}$ & 18,09 & $\frac{449,88 \pm 16,73}{331-615}$ & 18,59 & 97,0 \\
\cline { 2 - 7 } & 2014 & $\frac{296,60 \pm 15,87}{120-408}$ & 26,75 & $\frac{283,80 \pm 16,12}{116-401}$ & 28,41 & 95,7 \\
\hline \multirow{2}{*}{$\mathbf{4}$} & 2013 & $\frac{456,20 \pm 17,76}{336-600}$ & 19,47 & $\frac{441,76 \pm 17,27}{325-598}$ & 19,54 & 96,8 \\
\hline
\end{tabular}

The coefficient of seed productivity in nature varies between 85,2 and 93,1\%, under cultivation - between 94,0 and 96,6\%. P. vulgaris individuals studied by Yu. Kurochkina (2015) in Novosibirsk Oblast are characterized by somewhat lesser values of productivity (Cp. does not exceed 78,7\%). A significant coefficient of productivity $(90 \%)$ is recorded for southern Tomsk Oblast (90\%) [8]. High reproductive indicators of $P$. vulgaris cause anxiety at foreign scientists. In evergreen tropical forests of South America the species is evident as invasive, it easily spreads and brings the threat to local flora [12].

Germinating ability and germination energy of erems 6 months after gathering are higher compared to germinating ability of erems kept for 2 years. Percentage of germination is usually higher in erems collected in true meadows. The optimal temperature for germination turns out to be $28-30^{\circ} \mathrm{C}$. At such temperature regime germinating ability of seeds after 6 months of storage varies between 62,5 and 90,5\%, 2 years after gathering between $30,0 \%$ and $69,5 \%$. As the temperature regime decreases, germination indices 
decline reaching the minimum at $10-13^{\circ} \mathrm{C}$. Germinating ability of seeds collected in culture is somewhat higher (up to $97,5 \%$ ) than in nature. Germination energy is higher $(96,5 \%$ on the 10 th day of germination).

Biological productivity of $P$. vulgaris individuals in culture is higher in comparison with that in their natural habitats. Individuals at ripe generative ontogenetic stage are characterized by the greatest above-ground mass at sowing density of 100 seeds per 1 running meter. The above-ground mass of raw material obtained under introduction during the second year of vegetation $(228,05 \pm 36,05 \mathrm{~g} / \mathrm{run} . \mathrm{m})$ exceeds dry mass of raw material collected in the wild by $5-10$ times $\left(\mathrm{g}_{2}-3,17 \mathrm{~g} / \mathrm{m}^{2}\right)$.

\section{Conclusion}

$P$. vulgaris is a species of good potential ability of propagation by seeds. In various ecological-phytocoenotic conditions the potential seed productivity of individuals varies from 85,4 it. to 160,3 it., the real one - from 73,6 it. to 149,2 it.; germination ability of erems under optimal conditions of germination varies between 62,5 and $90,5 \%$. Emergence of plantlets in the wild has irregular character and depends on weather conditions of the current year. Under introduction the indices of seed productivity increase by 2-5 times, germination of erems - not more than by 1,5 times.

Biological productivity of $P$. vulgaris individuals in culture is by 5-10 times higher compared to that in their natural habitats $\left(g_{2}-3,17 \mathrm{~g} / \mathrm{m}^{2}\right)$. Individuals of the second year of development at the ripe generative ontogenetic stage are characterized by the greatest above-ground mass $(228,05 \pm 36,05 \mathrm{~g} /$ run.m)

The study was conducted within the framework of the Project of State Assignment № AAAA-A17117012610053-9 (CSBG SB RAS, Russia).

\section{References}

1. A.G. Borisova, Flora SSSR 20 (Academiae Scientiarum, Moscow, 1954)

2. Vegetable resources of Russia. Wild flowering plants, their component composition and biological activity. Families Caprifoliaceae - Lobeliaceae 4 (KMK, St. Petersburg, 2011)

3. T.E. Miller, A.A. Winn, D.W. Schemske, Amer.J.Bot. 81, 1-6 (1994)

4. A.A. Winn, Ecology 73, 831-840 (1985)

5. A.A. Winn, P.A. Werner, Ecology 68, 1224-1233 (1987)

6. A.A. Winn, Ecology 69, 1537-1544 (1988)

7. N.Yu. Kurochkina, Bul. of ASAU 11, 84-87 (2015)

8. T.G. Harina, N. V. Shvydkaya, Tomsk oblast nature complex 2 (TGU, Tomsk, 1995)

9. I.V. Vaynagiy, Bot. J. 59, 826-831 (1974)

10. M.G. Nikolaeva, M.V. Rasumova, V.N. Gladkova, Reference book on dormant seed germination (Nauka, Leningrad, 1985)

11. J. Jantunen, K. Saarinen, A. Valtonen, S. Saarnio, Applied Vegetation Science 10, 285-292 (2007)

12. O. Godoy, A. Saldana, N. Fuentes, F. Valladares, E. Gianoli, Biol.Inv. 13, 1615-1625 (2011). 